REVISTA

Actualidades Investigativas en Educación http://revista.inie.ucr.ac.cr/

ISSN 1409-4703

ISSN $1409-4703$

\title{
SISTEMATIZACIÓN TRABAJOS DE GRADO: PROPUESTA INVESTIGATIVA PARA LA RECONSTRUCCIÓN DE RUTAS DE CONOCIMIENTO
}

SYSTEMATIZATION OF DEGREE WORKS: RESEARCH PROPOSAL FOR RECONSTRUCTION OF ROUTE KNOWLEDGE

\author{
Volumen 15, Número 2 \\ Mayo - Agosto \\ pp. $1-20$
}

Este número se publicó el $1^{\circ}$ de mayo de 2015

DOI: http://dx.doi.org/10.15517/aie.v15i2.18967

Rubinsten Hernández Barbosa

Claudia Patricia Orjuela José Cabrera Paz

Luis Miguel Cabrera González

Revista indizada en REDALYC, $\underline{\text { SCIELO }}$

Revista distribuida en las bases de datos:

CATÁLOGO DE LATINDEX, IRESIE, CLASE, DIALNET, DOAJ, E-REVIST@S, SHERPA/ROMEO, QUALIS, MIAR

Revista registrada en los directorios:

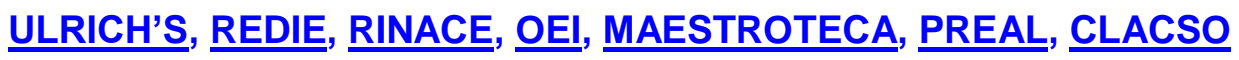




\title{
SISTEMATIZACIÓN TRABAJOS DE GRADO: PROPUESTA INVESTIGATIVA PARA LA RECONSTRUCCIÓN DE RUTAS DE CONOCIMIENTO \\ SYSTEMATIZATION OF DEGREE WORKS: RESEARCH PROPOSAL FOR RECONSTRUCTION OF ROUTE KNOWLEDGE
}

\author{
Rubinsten Hernández Barbosa ${ }^{1}$ \\ Claudia Patricia Orjuela ${ }^{2}$ \\ José Cabrera Paz \\ Luis Miguel Cabrera González ${ }^{4}$
}

Resumen: En este artículo se exponen, de manera sucinta, aspectos del diseño y planeación de un proyecto de investigación, cuya finalidad es sistematizar los trabajos de grado de la Maestría en Didáctica de las Ciencias de la Universidad Autónoma de Colombia. Esta investigación realizará una revisión documental a partir de categorías de análisis, instrumentos de mapeo, matrices y rutas de conocimiento. El texto está dividido en cuatro partes: la primera, referida a las consideraciones teóricas, las cuales abordan el objeto de estudio bajo tres lineamientos: sistematización de la base de conocimiento, análisis del posible impacto y la red de egresados. La segunda, establece, de manera general, la ruta metodológica que se ha considerado y la tercera, describe algunos resultados de las acciones al interior del equipo de trabajo. Finalmente, en la cuarta parte, se resalta el propio proceso investigativo y su importancia en el marco del proyecto curricular.

Palabras clave: AUTOEVALUACIÓN, COMUNIDAD DE PRÁCTICA, PROYECTOS DE INVESTIGACIÓN, RED ACADÉMICA, SISTEMATIZACIÓN, COLOMBIA

\begin{abstract}
This article presents in a concise manner, aspects of the design and planning of a research project whose aim is to systematize the work of the Master's degree in Science Education of the "Universidad Autónoma de Colombia". This research will conduct a documentary revision from categories of analysis, mapping tools, matrix and route knowledge. The text is divided into four parts: the first one, based on theoretical considerations, which address the subject of study under three guidelines: systematization of the knowledge base, analysis of the impact and the alumni network. The second one sets, in general, the methodological route. The third one describes some results of the actions within the teamwork. Finally, in the fourth part, is aimed at highlighting the research process itself and its importance within the curriculum project.
\end{abstract}

Keywords: ACADEMIC NETWORK, COMMUNITY OF PRACTICE, RESEARCH PROJECTS, SELFEVALUATION, SYSTEMATIZATION, COLOMBIA

\footnotetext{
Docente investigador, Universidad Autónoma de Colombia. Doctorado Universidad Distrital "Francisco José De Caldas. Dirección electrónica: rhbjd@hotmail.com

2 Docente investigador, Universidad Autónoma de Colombia. Magíster En Docencia de Las Matemáticas. Dirección electrónica: Claudia.orjuela@fuac.edu.co

${ }^{3}$ Investigador Psicólogo Experto en Aprendizaje y Tecnología Digital, Universidad Autónoma de Colombia. Dirección electrónica: cabrerapaz@yahoo.com

4 Docente investigador, Universidad Autónoma de Colombia. Magister en Educación a Distancia. Dirección electrónica: cabreralm@gmail.com
}

Artículo recibido: 3 de junio, 2014

Enviado a corrección: 19 de octubre, 2014

Aprobado: 20 de abril, 2015 


\section{Introducción}

Las tesis, monografías y prácticas formativas son objetos, a la vez que procesos de conocimiento que deben mostrar nuevas dinámicas. No solamente son objetos del ritual de finalización de un ciclo formativo, sino que, en sí mismos, pueden mostrarnos toda una "radiografía" de cómo los sujetos sociales, quienes desarrollan un programa formal de formación, están procesando conocimiento, construyendo rutas de indagación y recreando su aprendizaje en relaciones diversas con sus prácticas sociales. El proyecto de investigación que actualmente se desarrolla y que dio origen a este texto tiene este telón de fondo, pues al indagar sobre estos objetos de conocimiento y su estructura, los trabajos de grado de los estudiantes también dan cuenta, a través del propio texto, de cómo en la universidad contemporánea una de sus principales funciones, la investigación, se está desarrollando. En ese sentido, resulta claro que se pueden establecer tensiones en el conocimiento mismo generado, en la ruta establecida para su desarrollo y en la centralidad misma que tiene para el sujeto social y su relación con las realidades del entorno.

Desde su creación, el programa de la Maestría en Didáctica de las Ciencias de la Universidad Autónoma de Colombia ha consolidado un equipo de trabajo centrado en el campo de la Didáctica de las Ciencias y en el uso didáctico de las herramientas tecnológicas, generando un cúmulo de conocimiento a través de los trabajos de grado de los estudiantes del programa. En el desarrollo de este proceso se ha construido una dinámica y un conjunto de producciones cuya complejidad, alcances y sentido requieren ser estructurados, monitoreados, fortalecidos y dados a conocer a la comunidad de educadores en ciencias.

Al considerar que es mediante el trabajo de los estudiantes que un proyecto académico cobra vida, específicamente a través de las propuestas investigativas, las cuales se consolidan y fundamentan en el proceso de investigación, se establece la necesidad de analizar a la luz de unas categorías que permitan establecer con mayor claridad cómo se construye dicho proceso, qué tanto se fundamenta en su formación, por cuáles rutas de conocimiento transcurre, desde qué horizonte de preocupaciones e intereses se formula y, especialmente, a cuál necesidad estratégica del escenario educativo, pedagógico y didáctico corresponde.

Los trabajos de grado se constituyen en un elemento que consolida el proceso del estudiante-docente-investigador, inscrito en las líneas de investigación de la maestría. En estos trabajos se hace explícito; entre otras cosas, la manera en que se ha gestionado el conocimiento para los estudiantes (Baets, 2010). Pero también, para los docentes y el 
cuerpo administrativo, además, permite establecer criterios de evaluación de aspectos que el programa necesita revisar constantemente y que se relaciona con sus procesos investigativos, metodológicos, de aplicación e impacto en el campo de la Didáctica de las Ciencias.

Este proyecto busca, entre otros elementos, hacer explícito, recuperar y organizar las rutas por las cuales ha transcurrido el desarrollo de este proceso de conocimiento en el plano institucional. Con ellas se puede establecer, al menos en ciertos aspectos cruciales fundamentados en la historia de los desarrollos del programa- las bases para el diseño y la formulación institucional de líneas de investigación. En ese sentido, el recorrido que ha hecho la propia comunidad permite mantener una sintonía fundamental con problemáticas, preguntas y formas de trabajo que los propios estudiantes han realizado.

Este proyecto también permitirá evidenciar los temas de investigación del trabajo de grado de los estudiantes, las maneras de formular y gestionar sus proyectos, los anclajes a sus instituciones y contextos, los cuales son un vínculo con la realidad investigativa, social y educativa del país, aspecto que necesita ser develado, no solo por su función epistemológica, investigativa o práctica, sino por el sentido del compromiso que debe tener la relación universidad-país. Habida cuenta del valor de la universidad como gestora del cambio social, nada es más útil para ello que el conocimiento producido por los organismos especializados, abierto y puesto en la escena pública, aprovechando el poder de difusión del mundo digital.

En el mismo sentido, el desarrollo de este proyecto no solo apuesta por la sistematización, reconstrucción de las líneas de investigación y los procesos de desarrollo del conocimiento por parte de los estudiantes de la Maestría en Didáctica de las Ciencias, sino que apunta a proveer al programa una estrategia metodológica para compartir sus saberes, pues la universidad es una de las instituciones más importantes de la sociedad; generadora de información y conocimiento, que debe apostar por compartir su acumulado de producciones con las diversas comunidades, potenciales usuarios y beneficiarios.

Teniendo como referente el proceso de sistematización de los trabajos de grado, se pretende elaborar una propuesta metodológica para construir un banco de conocimiento digital on line (en línea), abierto a los docentes interesados en el plano interno (profesorado de la universidad y estudiantes de la maestría), así como a todos aquellos interesados en profundizar o crear proyectos investigativos que giren alrededor de los temas y ámbitos de investigación que se han desarrollado en las dos líneas que aparecen institucionalizadas. 
En síntesis, el proyecto tiene como objetivo analizar, describir y sistematizar las tesis de Maestría en Didáctica de las Ciencias de la Universidad Autónoma de Colombia, requisito de grado del programa desde la primera cohorte hasta la fecha ${ }^{5}$. El trabajo de investigación descrito en este documento busca realizar una revisión documental, con el fin de organizar los productos de investigación desde varias categorías de análisis, con instrumentos de mapeo, matrices y rutas de conocimiento. También, se pretende determinar el impacto que los trabajos de grado de la maestría han tenido en el desempeño profesional de sus egresados. Finalmente, se busca canalizar el talento humano generado para construir una red de egresados, que a manera de comunidad de práctica y con un banco de conocimiento digital, permita la permanente vinculación del programa con sus profesionales egresados en Didáctica de las Ciencias, quienes apoyen el crecimiento y cualificación del proceso formativo e investigativo de la maestría.

\section{Consideraciones teóricas}

En esta propuesta investigativa se conjugan tres dimensiones: la primera; sistematización de la información que conforma una base de datos de conocimientos. Esta pretende determinar la conceptualización sobre los procesos subyacentes en la construcción y desarrollo de los proyectos de investigación de los estudiantes. La segunda; análisis del impacto. Busca establecer cuál ha sido el impacto de la formación en el desarrollo profesional de los egresados y, la tercera; red de egresados. Procura consolidar una red académica en el campo de la Didáctica de las Ciencias. Por ello, se considera que una comunidad, a partir de los ejes de sistematización emergentes descritos en este texto, debe formular y fomentar la puesta en marcha de un proceso de red entre los diferentes componentes del sistema de la maestría, con el fin de estimular un mejor proceso de vinculación permanente, de creación de estrategias de comunidad de formación que posibiliten generar redes de relación efectivas entre el programa, la universidad y sus egresados. Seguidamente, se procederá a describir las tres dimensiones.

\footnotetext{
${ }^{5}$ La maestría en Didáctica de las Ciencias empieza a funcionar en el segundo semestre del 2008 y los primeros estudiantes que se graduaron lo hicieron en el segundo semestre del 2010. A Diciembre de 2014 se han graduado 48 estudiantes.
} 


\subsection{Sistematización de la base de conocimientos}

Abordar el proceso de analizar, describir, sistematizar y construir una base de conocimientos a partir de los trabajos de grado, a fin de establecer en ellos los mapas de conocimiento, las líneas de continuidad o ruptura, las estrategias, campos argumentales, objetos y formas metodológicas. Este proceso de análisis se constituye en un objeto de conocimiento, el cual se inscribe en una práctica investigativa. Por ello, se concibe el proceso investigativo como una construcción dinámica que da cuenta del proceso evolutivo de los productos investigativos durante el proceso formativo del programa de la maestría.

En este sentido, indagar, analizar y organizar un sistema de objetos de conocimiento (Geisler et al., 2007) como los trabajos de tesis, producto de un proceso de indagación, conlleva también una pregunta fundamental debido a la formación investigativa. En este aspecto, el desarrollo analítico se relaciona con la necesidad de vincular la investigación con la formación y, a partir de ese eje argumentativo, generar una indagación que permita conocer cómo se construye la articulación desde una práctica, estructura y dinámica del objeto que la hace visible; es decir, el trabajo de tesis. En esta dimensión es importante considerar dos factores:

\subsubsection{Objetos de conocimiento: proyectos de grado}

Cada proyecto de grado construido y reconstruido por los estudiantes de la maestría, con la ayuda de los docentes del programa y directores de tesis durante su proceso de formación, establecidos paralelamente a la fundamentación teórica, van decantando su proyecto de tesis. Este ejercicio es para el estudiante una experiencia de proceso investigativo y de aprendizaje sistemático. Aprender en la universidad significa construir la experiencia del conocimiento en cada sujeto del proceso formativo (Thomas y Seely, 2011). En este sentido, la acción formativa genera en el sujeto de aprendizaje una experiencia singular de construcción: no hay adquisición de conocimiento, sino una experiencia de construcción cognitiva (Jong et al., 2009; Blakemore, 2010). Un proyecto de grado es un cruce de conocimientos entre la construcción personal e institucional, lo cual se evidencia como objeto de conocimiento público.

De esta manera, el proyecto de grado es un producto formativo, donde la investigación en la formación significa la experiencia que cada sujeto hace frente al mundo cuando construye conocimiento sobre éste. Conocer es construir, construir conocimiento es indagar el mundo del sujeto. Esto nos lleva a identificar una línea de continuidad entre procesos 
cognitivos en la investigación y procesos de aprendizaje en la formación. Deslindar uno del otro, aún en el análisis y sistematización de los proyectos de grado es complejo y no menos que artificial. En cualquiera de las dos dimensiones, el sujeto construye conocimiento significativo frente a una determinada demanda social, ya sea en la actividad formativa 0 investigativa.

La investigación como práctica de conocimiento sistemática, evidenciada en un trabajo de grado, es una estrategia formativa, articulada de diversas maneras al desarrollo disciplinar y profesional de la universidad. Una formación de carácter investigativo entiende que el sujeto en la acción formativa es un ser que construye conocimiento de diversas maneras y por distintas rutas de argumentación, a la vez que considera el conocimiento como una experiencia multidimensional que articula lo que el sujeto sabe con la responsabilidad por lo que hace con su saber (Mclntosh, 2010). En cada trabajo de grado se puede evidenciar esa ruta, su dinámica y por supuesto, su potencial, para luego confrontarlo con el análisis del impacto de la formación en el campo profesional de los propios estudiantes y de las instituciones y comunidades a las que pertenece.

Vale la pena mencionar que, para el caso particular de la Maestría en Didáctica de las Ciencias de la Universidad Autónoma de Colombia, el proyecto de investigación de los estudiantes surge de sus intereses particulares, los cuales se circunscriben en las problemáticas identificadas en su quehacer profesional ${ }^{6}$. Es como si se preguntara qué se construyó versus qué se ha desarrollado en el marco institucional y profesional del estudiante de dicha maestría. Por eso, este proceso de sistematización está orientado a la necesidad de conocer el impacto de la formación en el contexto profesional del estudiante, para lo cual cobran sentido preguntas como: ¿qué tan eficaz fue esta práctica investigativa?, ¿posibilitó nuevos abordajes, ejercicios, problemas y tematizaciones?, ¿de qué manera aportó a las transformaciones en el aula?, ¿cuáles fueron sus aportes al campo de la didáctica y sus disciplinas? Estas interrogantes implican asumir que el conocimiento es multidimensional; tiene matices y ángulos diversos, rutas de construcción distintas y formas de asociar una categoría y eficacia institucional también diversa (Visser y Visser, 2010).

De lo anterior, emerge una inquietud, ¿cómo son los objetos que construyeron los estudiantes y cómo fue su posterior desarrollo? Esta pregunta articula el proceso de

\footnotetext{
${ }^{6}$ Uno de los requisitos que tiene el programa de Maestría, y que es requisito para ser admitido como estudiante regular, es que el docente presente una propuesta de investigación. Cuando se indaga por la génesis de ésta, se evidencia que surge de su propia práctica pedagógica y que necesita ser abordado para el mejoramiento de la misma.
} 
sistematizar los trabajos de grado con el impacto que se puede vislumbrar en los procesos profesionales de los egresados. La pertinencia de esta pregunta se inscribe en el más amplio espectro del valor de la formación posgradual: el conocimiento está situado en comunidades locales y allí opera como un potencial transformador.

Esto constituye un matiz de alto valor del cuestionamiento, máxime cuando la mayor parte de los egresados tienen su desempeño en el sistema educativo básico y medio y se requiere que la formación docente tenga desarrollos de mejoramiento de sus prácticas profesionales en contexto. En este sentido, esta investigación no solo valorará los "objetos de conocimiento construidos" (o sea los trabajos de grado), sino cómo este proceso se evidencia, hasta dónde llega y cuánto de su valor es posible observar en el desarrollo profesional y en la transformación de las prácticas de los egresados de la maestría.

Teniendo como referente lo expuesto en los párrafos anteriores, el equipo de trabajo considera que un modelo de gestión del conocimiento es conceptualmente funcional para valorar la gestión de la investigación en las organizaciones universitarias, dado que permite el desarrollo de un abordaje que articula los bienes tangibles con los intangibles en los procesos que en ella se desarrollan. El conocimiento ya no está para ser almacenado en las reservas de las organizaciones, sino que debe ser un "flujo permanente" (Hagel, Seely Brown y Davison, 2010), dispuesto a ser aprovechado, disponible para alimentar la propia dinámica y construcción cotidiana de la organización.

Un referente básico del modelo de gestión del conocimiento se puede componer de al menos tres elementos básicos (Bueno, 2003):

- El capital humano: se refiere al conocimiento que tienen las personas, habilidades, talentos y destrezas. Este capital puede ser desarrollado a través de experiencias de aprendizaje pertinentes y permanentes.

- El capital estructural: constituido por los soportes externos que posee una organización para desarrollar su trabajo. Los sistemas de información, mecanismos de gestión y bibliografía -entre otros- son ejemplos del capital estructural.

- El capital relacional: hace referencia a los diferentes tipos de vínculos investigativos, formativos y financieros que la organización tiene con el entorno y sus diferentes actores sociales (Bueno, 2003).

Para desarrollar la gestión de las estrategias de investigación, este modelo permite diferenciar las que pertenecen a lo fundamental de un proceso de conocimiento: el capital 
humano, lo que corresponde a la materialidad. Es decir, la base estructural, que funciona a manera de "medios" para la investigación y que alude a la capacidad de interacción del sistema investigativo con el entorno.

En otras palabras, el abordaje de referencia de gestión del conocimiento y capital intelectual pasa por una articulación de sujetos, interacciones, medios y procesos de "construcción de conocimiento profesional" (Bartholomew, 2008). Todos estos elementos se articulan en función de su objeto de producción; el conocimiento, que para efectos de una institución de educación superior, gira en torno a la ejecución de procesos y objetos de aprendizaje y conocimiento (materializados; por ejemplo, en un trabajo de grado). Acá, la organización de las experiencias cognitivas, la producción de contenidos y el desarrollo de aplicaciones, entre otros aspectos, dependen de la capacidad de articular cada componente en pro del logro de los objetivos y mediante estrategias concretas en las cuales se establece y desarrolla cada componente del modelo.

Para el proceso de este proyecto de investigación el componente por abordar se concentrará en los trabajos de grado y en la formulación del modelo de comunidades de práctica con los egresados. No obstante, los demás componentes del abordaje están presentes, pues cada objeto funciona como parte de este ecosistema de conocimiento.

\subsubsection{La reconstrucción de las rutas de conocimiento}

La formación investigativa es una experiencia a la que se llega desde multiplicidad de experiencias cognitivas, de intereses y necesidades. No existe una manera única de formar el conocimiento o el desarrollo del espíritu científico. En efecto, al conocimiento diverso se llega de múltiples formas (Koshy, 2005; Mclntosh, 2010). Cada vez es más reconocido que, luego de transitado un amplio trecho de polémicas abiertas, no existe en el universo del saber metodologías universales que pudieran ser estandarizadas a todos los dominios de conocimiento; ni siquiera en un determinado dominio de conocimiento, menos aún en el inestable, diverso y cambiante mundo de las ciencias de lo social, de lo educativo y del vínculo humano.

La investigación que se produce en un proyecto de grado en la formación universitaria puede ser entendida como el desarrollo y consolidación de una actitud cognitiva sistemática, una forma de conceptuar, observar y proceder en un campo determinado frente a un conocimiento diverso al cual se llega por distintas rutas y procedimientos. Sin embargo, aún moviéndonos en espacios multiformes, con paradigmas de conocimiento que identifican, 
prescriben y delimitan formas de saber propias de cada campo disciplinar, es necesario establecer cuáles fueron los propósitos comunes que orientaron el desarrollo de la formación investigativa y cómo estos se evidencian desde los propios objetos y argumentaciones que circularon por unas rutas de conocimiento que se espera identificar y problematizar.

En el análisis de esta base de conocimiento generado, el establecimiento de estas rutas permite reconstruir un mapa con líneas cognitivas, tendencias y horizontes de desarrollo. Los problemas que han guiado la indagación plantean problemáticas analíticas diversas. ¿Cómo se ha conectado lo que preguntan los egresados en sus trabajos de grado con sus contextos, cómo las preguntas tienen una relevancia frente al campo mismo de las comunidades de expertos, y a la vez, cómo esto plantea un diálogo de múltiples entradas con interlocuciones y tendencias argumentales de diversos campos? Habida cuenta de los perfiles de los estudiantes, cuya dimensión sociodemográfica habrá que caracterizar, se estima de entrada que su campo, el de la educación, es probablemente el que más problematizaciones y preguntas ha generado. Esto implica rastrear los escenarios visibles, cómo cada ruta de conocimiento lo hace explícito y lo aborda en su estrategia investigativa (pregunta, objetivos y metodologías, entre otros aspectos).

Se espera que esta ruta de conocimiento sea una manera de plantear los itinerarios realizados, para que desde su dinámica emergente se pueda alimentar y reformular -si es preciso- orientaciones sobre las formas y las estructuras investigativas que ha establecido la maestría. Estableciendo esta base de conocimiento, se hará explicito la congruencia entre las orientaciones, las rutas propuestas y las trayectorias desarrolladas por los procesos de conocimiento de los estudiantes.

\subsection{Análisis de impacto}

Analizar el impacto de un proceso de formación tiene dimensiones complejas y multidimensionales, siendo difícil deslindarlo del origen que ha generado dicho proceso. Sin embargo, apoyándonos en el proceso mismo de los egresados y en su propia capacidad autoevaluativa (Jong et al., 2009), se espera identificar las rutas de desarrollo y los derivados de la formación. Desde el enfoque del compromiso del conocimiento con el contexto social, los procesos de la universidad están orientados hacia la construcción de proyectos, estrategias y programas de investigación pertinentes que den alternativas de solución a problemáticas de contextos locales y globales, con los cuales interactúa dicha entidad. Vale anotar que en el universo de la interdependencia social, económica, científica y tecnológica 
(Page, 2008, Pagel 2012), las categorías local-global guardan líneas de continuidad e interacción, dentro de las cuales la investigación se ve obligada a plantear soluciones de aplicación local con carácter universal o viceversa. El desarrollo de la investigación en la universidad se constituye en la respuesta más efectiva de la formación hacia su sociedad. La investigación formativa y aplicada, evidente en los trabajos de grado, es entonces uno de los puentes más privilegiados y eficaces para construir una estrecha relación entre universidad y sociedad.

La institución asume que el conocimiento es, en la sociedad contemporánea, el capital social más estratégico para generar crecimiento material, bienestar humano y desarrollo responsable con el medio ambiente. En una sociedad como la nuestra, con fuertes problemas de inequidad en la distribución social de la riqueza, déficit en la generación de adecuados procesos de convivencia y bienestar colectivo, crecientes problemas ambientales y el desarrollo de una adecuada formación profesional, entre otros, resulta necesaria una mirada constante por el impacto de los egresados en sus nichos profesionales y su capacidad de adaptación al cambio.

Por ello, el análisis de impacto que complementa esta propuesta investigativa pretende mapear posibles formas de conectar formación con transformación de manera eficaz. Sin duda, subyace a este propósito la idea de organizar el proceso formativo, la práctica investigativa con la pertinencia y las exigencias que el propio contexto del egresado genere. Un programa de maestría que dialogue con las necesidades del egresado tiene mayor flexibilidad y puede adaptarse mejor, no solo al proceso formativo formal, sino a todo el potencial de formación de actualización y cualificación de formación que puede requerir el egresado mismo una vez titulado.

\subsection{La red de egresados}

A partir del proceso de investigación y el desarrollo de la indagación por el impacto del proceso formativo, este proyecto apuntará a fomentar una comunidad de práctica (Blackmore, 2010) formulada como red de egresados. En este diseño, se espera generar un vínculo más estrecho entre el programa, el proceso de conocimiento y la creciente comunidad de profesionales formados en los posgrados en educación. Esto tiene múltiples implicaciones, pero el diseño espera conceptualizar los desarrollos para explorar qué alcances y resultados pueden generarse en un proceso de red cognitiva (Mahmoud, 2007). 
Sin duda, una universidad en constante y efectiva comunicación con los egresados ve en las redes el potencial para estar conectada con sus profesionales y construir, de manera colectiva, una comunidad académica, en este caso, en torno a la Didáctica de las ciencias. En esta medida, el terminar la formación de la maestría no es el fin de un proceso, sino el punto de partida para considerar un papel activo y propositivo en torno a la construcción de nuevos conocimientos en las dos líneas de investigación del programa.

Lo anterior, no solo tiene un propósito de crecimiento para el programa mismo en su dimensión formativa e investigativa, sino que permite crear una comunidad potencialmente de beneficio mutuo que facilite a los actores involucrados un crecimiento y aprovechamiento del sentido de actuar en red. De hecho, la lógica de red y las comunidades de práctica se están volviendo un proceso clave de la sociedad del conocimiento (Castells, 2005; Bingham y Conner, 2010), orientadas hacia la posibilidad de crear nuevos nichos de gestión de aprendizajes y conocimiento, con el fin de crear una universidad más conectada con su entorno.

La formulación de una red de egresados, desde una estrategia de generación de comunidad de aprendizaje, parte de reconocer el valor esencial de la construcción colectiva del conocimiento, así como el valor de la construcción organizada de una red de trabajo que conecte los contextos de los egresados. En este sentido, en la medida en que la sociedad contemporánea ha desbordado y traspasado fronteras de saber, la universidad está orientada a hacer de la gestión de conocimiento investigativo una práctica que articule dominios disciplinares, metodologías, comunidades y diversos contextos profesionales.

Generar una comunidad de práctica con los egresados tiene para la maestría en Didáctica de las Ciencias y, en general, para los postgrados en educación, dos dimensiones básicas: la primera; el reconocimiento de que el saber humano es un constructo colectivo, que mediante la organización de un sistema dinámico de comunicación con entornos diversos es posible generar nichos sostenibles y estructurados de conocimiento renovadas de manera permanente. Responder qué tan conectado está un programa con su contexto y lo canales de comunicación que utiliza, permite generar pertinencia, sentido, profundidad y horizonte de trabajo estratégico. Eso puede venir, sin duda, de los propios egresados, que funcionan como uno de los mejores mecanismos para conectar postgrado-educacióncontexto. Una comunidad de práctica, a su vez, implica compartir talentos, los que en buena medida el programa ha contribuido a desarrollar y construir sobre la pluralidad, las 
diferencias de comprensión y abordaje del mundo simbólico y material en el que los propios egresados se mueven.

\section{Posible ruta metodológica}

Teniendo en cuenta los objetivos de la investigación, se propuso una metodología de corte descriptivo-interpretativo (Hernández, 2003). A continuación, se describen las fases del proyecto y las actividades que se realizarán con miras a la obtención de los objetivos propuestos. En el siguiente cuadro, se establece la posible ruta metodológica que los autores han considerado y que se organiza en fases, actividades, objetivos y productos esperados. Estos aspectos se sintetizan en la tabla 1.

Tabla 1. Posible ruta metodológica

\begin{tabular}{|c|c|c|c|}
\hline Fases & Objetivo & Actividades & Productos esperados \\
\hline $\begin{array}{l}\text { Revisión } \\
\text { documental }\end{array}$ & $\begin{array}{l}\text { Organizar y } \\
\text { categorizar los } \\
\text { trabajos inicialmente } \\
\text { basados en las dos } \\
\text { líneas de investigación } \\
\text { con las que cuenta el } \\
\text { programa de maestría. }\end{array}$ & $\begin{array}{l}\text { Lectura de los } \\
\text { resúmenes } \\
\text { introducción de cada } \\
\text { uno de los trabajos } \\
\text { de grado. }\end{array}$ & $\begin{array}{l}\text { Organización de los } \\
\text { documentos. } \\
\text { Identificación de las } \\
\text { temáticas abordadas en los } \\
\text { trabajos. } \\
\text { Visualización de las } \\
\text { problemáticas } \\
\text { generaron la investigación. } \\
\text { Caracterización de las rutas } \\
\text { metodológicas que fueron } \\
\text { abordadas en los trabajos } \\
\text { de investigación. }\end{array}$ \\
\hline $\begin{array}{l}\text { Elaboración del } \\
\text { Instrumento de } \\
\text { categorización } \\
\text { de los trabajos } \\
\text { de grado }\end{array}$ & $\begin{array}{l}\text { Caracterizar } \text { los } \\
\text { trabajos de grado de } \\
\text { acuerdo con los } \\
\text { aspectos establecidos } \\
\text { en el instrumento } \\
\text { diseñado para tal fin. }\end{array}$ & 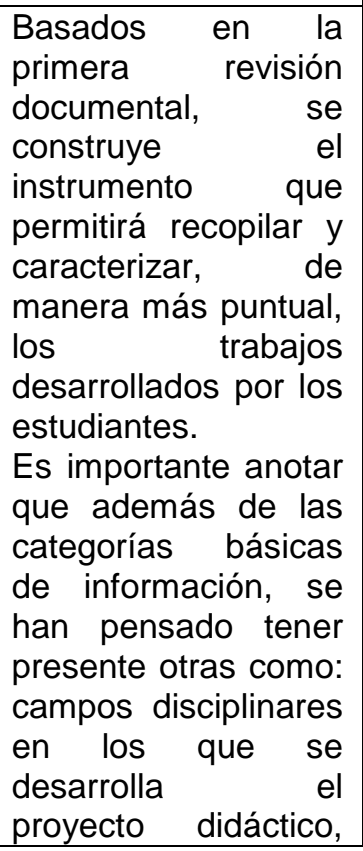 & $\begin{array}{l}\text { Construcción de un matriz, } \\
\text { inicialmente propuesta por } \\
\text { los investigadores, y luego } \\
\text { valorada y validada por } \\
\text { expertos investigadores. } \\
\text { También se considera la } \\
\text { participación interna de } \\
\text { docentes y egresados de la } \\
\text { propia maestría. } \\
\text { Una vez organizada la } \\
\text { información, se reestructura } \\
\text { el instrumento y de nuevo se } \\
\text { pone a discusión por el } \\
\text { equipo de trabajo, para } \\
\text { hacer un ejercicio piloto con } \\
\text { un trabajo de tesis, el cual } \\
\text { servirá de modelo para el } \\
\text { instrumento final, que será } \\
\text { aplicado a todos los trabajos } \\
\text { de grado. }\end{array}$ \\
\hline
\end{tabular}




\begin{tabular}{|c|c|c|c|}
\hline & & $\begin{array}{l}\text { nivel educativo en } \\
\text { que se desarrolla el } \\
\text { proyecto, tipos de } \\
\text { participantes/fuentes } \\
\text { que participaron } \\
\text { como sujetos y/o } \\
\text { objetos del estudio, } \\
\text { enfoque conceptual y } \\
\text { metodológico, clases } \\
\text { instrumentos y tipo } \\
\text { de fuentes, entre } \\
\text { otras. }\end{array}$ & \\
\hline $\begin{array}{l}\text { Análisis del } \\
\text { impacto }\end{array}$ & $\begin{array}{llr}\text { Establecer } & & \text { la } \\
\text { relevancia y el impacto } \\
\text { del programa } & \text { de } \\
\text { maestría en } & \text { el } \\
\text { desempeño } & & \\
\text { profesional de } & \text { los } \\
\text { egresados. } & & \\
\end{array}$ & 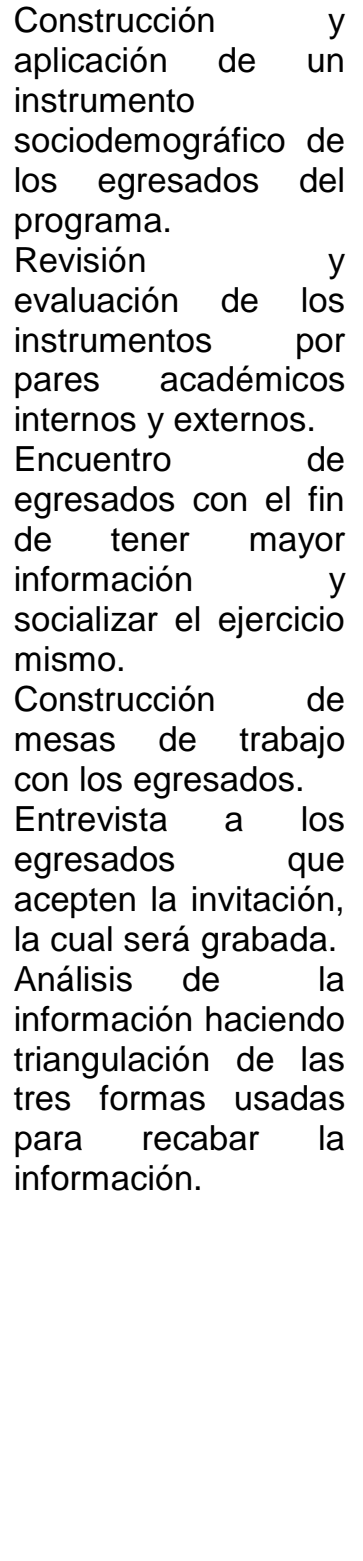 & 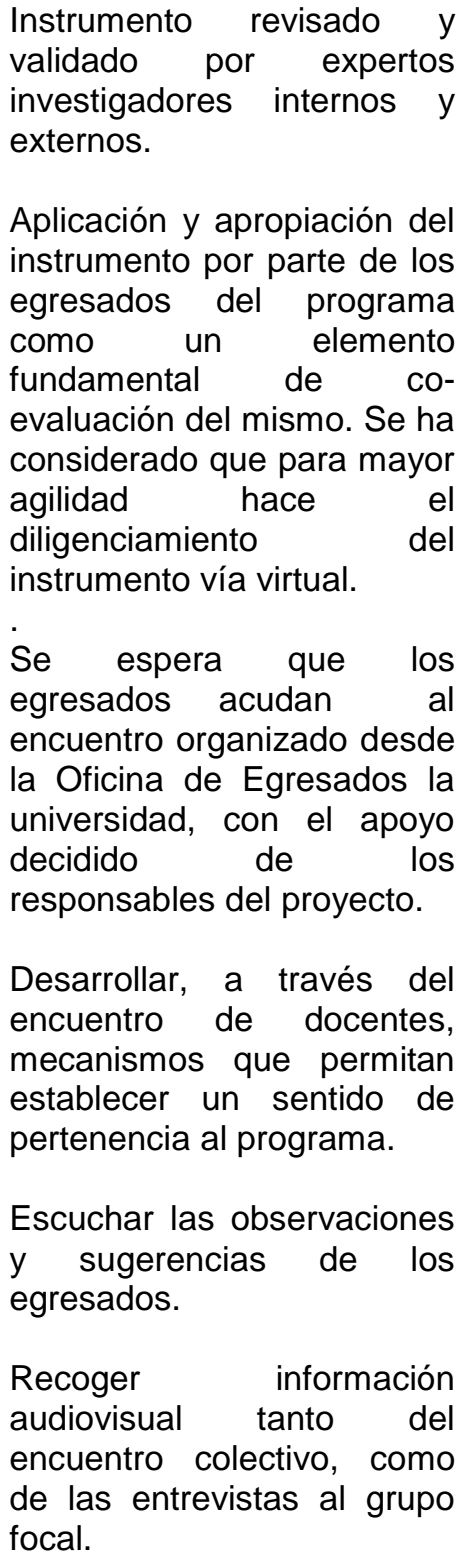 \\
\hline \begin{tabular}{lr}
\multicolumn{2}{l}{ Conformación } \\
de & red \\
académica & $y$
\end{tabular} & $\begin{array}{l}\text { Diseñar, desarrollar e } \\
\text { implementar el portal } \\
\text { de la Maestría en }\end{array}$ & $\begin{array}{l}\text { Construcción del } \\
\text { portal del programa } \\
\text { de } \\
\text { maestría. }\end{array}$ & $\begin{array}{l}\text { Se tiene la gran expectativa } \\
\text { de tener a disposición de los } \\
\text { estudiantes, docentes y los }\end{array}$ \\
\hline
\end{tabular}




\begin{tabular}{|c|c|c|c|}
\hline $\begin{array}{l}\text { Comunidad de } \\
\text { Práctica }\end{array}$ & $\begin{array}{l}\text { Didáctica de las } \\
\text { Ciencias de la } \\
\text { Universidad Autónoma } \\
\text { de Colombia. }\end{array}$ & $\begin{array}{lr}\text { Apertura del espacio } \\
\text { en el portal para la } \\
\text { socialización } \\
\text { retroalimentación de } \\
\text { experiencias } \\
\text { pedagógicas } \\
\text { investigativas. }\end{array}$ & $\begin{array}{l}\text { egresados el portal del } \\
\text { programa de Maestría en } \\
\text { Didáctica de las Ciencias de } \\
\text { la Universidad Autónoma de } \\
\text { Colombia. } \\
\text { Se espera conformar una } \\
\text { red de egresados que tenga } \\
\text { fundamento en la } \\
\text { comunidad de práctica } \\
\text { activa y participativa, } \\
\text { comprometida con el } \\
\text { desarrollo de la Didáctica de } \\
\text { las Ciencias. } \\
\text { También se tiene la } \\
\text { expectativa de que el portal } \\
\text { se convierta en un medio } \\
\text { activo de comunicación } \\
\text { entre todos los miembros de } \\
\text { la maestría, incluyendo los } \\
\text { egresados. Que el portal } \\
\text { sea el espacio para } \\
\text { compartir las experiencias } \\
\text { de aula, los grupos y } \\
\text { proyectos de investigación a } \\
\text { los que se encuentran } \\
\text { vinculados los egresados, } \\
\text { invitaciones a seminarios y } \\
\text { congresos tano nacionales } \\
\text { como internacionales, } \\
\text { también para saber sobre } \\
\text { premios y reconocimientos } \\
\text { que han tenido los } \\
\text { miembros de la maestría, } \\
\text { conocer y compartir las } \\
\text { publicaciones, en fin para } \\
\text { conformar una comunidad } \\
\text { de práctica activa. }\end{array}$ \\
\hline $\begin{array}{l}\text { Socialización de } \\
\text { los resultados } \\
\text { del proyecto }\end{array}$ & $\begin{array}{lr}\text { Socializar } & \text { los } \\
\text { resultados } & \text { del } \\
\text { proyecto a nivel local, } \\
\text { nacional } \\
\text { internacional. }\end{array}$ & $\begin{array}{lr}\text { Presentación de los } \\
\text { resultados a las } \\
\text { directivas y docentes } \\
\text { de la maestría, a los } \\
\text { estudiantes } \\
\text { egresado. Escritura } \\
\text { de dos artículos y } \\
\text { asistencia r a } \\
\text { congresos } \\
\text { encuentros y } \\
\text { educación superior. }\end{array}$ & $\begin{array}{l}\text { Se espera escribir dos } \\
\text { artículos en revistas de alto } \\
\text { impacto, que den cuenta de } \\
\text { la planeación y diseño } \\
\text { metodológico, como también } \\
\text { de los resultados. }\end{array}$ \\
\hline
\end{tabular}




\section{Avances y resultados parciales del proyecto}

A continuación, se describirán los avances que ha generado el proyecto hasta la actualidad, especialmente en el desarrollo de las actividades propuestas para las dos primeras fases. Se procedió a construir dos instrumentos, de los cuales uno se orienta a recabar la información de los documentos escritos; es decir, del trabajo de tesis. Este instrumento se organizó por categorías y en cada una de ellas se consideraron varios aspectos, tal y como se muestra en la tabla 2.

Tabla 2. Instrumento de análisis documental.

\begin{tabular}{|l|l|}
\hline Categoría de análisis & \multicolumn{1}{|c|}{ Aspectos que se consideran en cada categoría } \\
\hline Datos básicos & $\begin{array}{l}\text { Título del trabajo, línea de investigación en la que se inscribe, } \\
\text { número de autores, año de terminación. }\end{array}$ \\
\hline $\begin{array}{l}\text { Estructura del } \\
\text { documento }\end{array}$ & $\begin{array}{l}\text { Problema de investigación, campo disciplinar, nivel educativo en el } \\
\text { que se desarrolla el proyecto, participantes de la comunidad } \\
\text { educativa, problema de investigación, y desarrollo conceptual, } \\
\text { enfoque metodológico e instrumentos, entre otros aspectos. }\end{array}$ \\
\hline Aspectos didácticos & $\begin{array}{l}\text { Abordaje y delimitación de los aspectos didácticos; estrategias } \\
\text { didácticas. }\end{array}$ \\
\hline $\begin{array}{l}\text { Fuentes y referencias } \\
\text { bibliográficas }\end{array}$ & $\begin{array}{l}\text { Libros, revistas, publicaciones periódicas, fuentes en línea. Periodos } \\
\text { de años de las fuentes e idioma. }\end{array}$ \\
\hline
\end{tabular}

El otro instrumento tuvo por objetivo recoger información sobre los egresados, a fin de posibilitar al programa de maestría en el fomento de la construcción de una red de egresados, el análisis del impacto del proceso formativo y la identificación de las necesidades actuales profesional de nuestros exalumnos, por lo que se estructuró de la misma manera que el anterior. Seguidamente, la tabla 3.

Tabla 3. Categorías de análisis en el instrumento de egresados.

\begin{tabular}{|l|l|}
\hline \multicolumn{1}{|c|}{ Categoría } & \multicolumn{1}{|c|}{ Aspectos que se consideran en cada categoría } \\
\hline Datos básicos & Nombre, teléfonos, género, email, edad, títulos, año de graduación. \\
\hline Ubicación profesional & $\begin{array}{l}\text { Tipo de institución donde labora (pública, privada), cargo, nivel en el } \\
\text { que se desempeña y rango de ingresos. }\end{array}$ \\
\hline Desarrollo Profesional & $\begin{array}{l}\text { Aspectos sobre el nivel de aprovechamiento de la maestría en } \\
\text { diferentes aspectos, dificultades, apoyo, impacto del trabajo y tipo de } \\
\text { organización a la que pertenece. }\end{array}$ \\
\hline Producción académica & $\begin{array}{l}\text { Número de artículos, libros, participación en eventos como ponente, } \\
\text { productos digitales y distinciones. }\end{array}$ \\
\hline Expectativas & $\begin{array}{l}\text { Proyectos que están desarrollando y aportes a la comunidad de } \\
\text { egresados. }\end{array}$ \\
\hline
\end{tabular}


Ambos instrumentos fueron validados por los docentes del programa, expertos investigadores externos a la institución y cuatro egresados, quienes aceptaron la invitación de participar. A todos ellos se les envió un resumen del proyecto y el instrumento de valoración que se organizó en una matriz que incluyó lo siguiente: denominación de la categoría, contenido y pertinencia del indicador a su respectiva categoría y redacción del indicador. También se incluyó un espacio para que el evaluador hiciera sugerencias y posibles modificaciones. El instrumento de egresados se colocó en la web y se puede consultar en http://caldas.semilleros.net/lime/index.php/964925/lang-es.

Otro aspecto que cabe mencionar en este apartado se refiere a lo concerniente con el encuentro de egresados, pues desde un comienzo se determinó que este espacio, además de reencontrarse con los amigos y pasar un rato agradable, se usaría para recopilar información sobre el impacto, en diferentes aspectos, que el programa de maestría ha generado en los participantes. Para ello, se contrató a un presentador, quien animaba el evento y, a su vez, mediante un plan de trabajo previamente establecido indagaba, de manera indirecta, sobre los siguientes aspectos: beneficio de la investigación desarrollada, si el trabajo realizado lo continúa ejerciendo, si lo dio a conocer a sus compañeros de área, a la institución, dificultades en el proceso investigativo y de qué tipo (acompañamiento, tiempo, tutor, bibliografía, temática, recurso tecnológico incorporado en el trabajo). También, su impacto en posibles publicaciones: revistas, libros, capítulo de libro. Asistencia a seminarios y congresos, necesidades de cualificación de los egresados; comunicación con la maestría, invitación a cursos y, por supuesto, su opinión sobre la conformación de la red de egresados.

Entre conversaciones, música y una cena, se le solicitó a los egresados asistentes leer y desarrollar el taller que se muestra a continuación, el cual pretendió responder a la pregunta ¿qué ha significado, desde una visión retrospectiva, su formación en la maestría? Se destaca su receptividad, participación y colaboración, quienes, además, celebraron la idea del encuentro y expresaron su deseo de cooperación, intervención y aportación a la red de egresados.

También manifestaron su intención de colaborar activamente desde su ejercicio profesional, así como de compartir y visualizar los proyectos que han adelantado en sus instituciones escolares, pues lo ven como un camino para identificarse, acogerse y pertenecer a una red que valora y potencia el trabajo y los aportes que cada uno de sus integrantes han construido en y para la colectividad. Cabe destacar que este evento fue grabado para su posterior análisis. 


\section{UNIVERSIDAD AUTÓNOMA DE COLOMBIA \\ MAESTRIA EN DIDÁCTICA DE LAS CIENCIAS \\ ENCUENTRO DE GRESADOS \\ BIENVENIDOS}

Hoy, un buen día para nuestra comunidad y su memoria

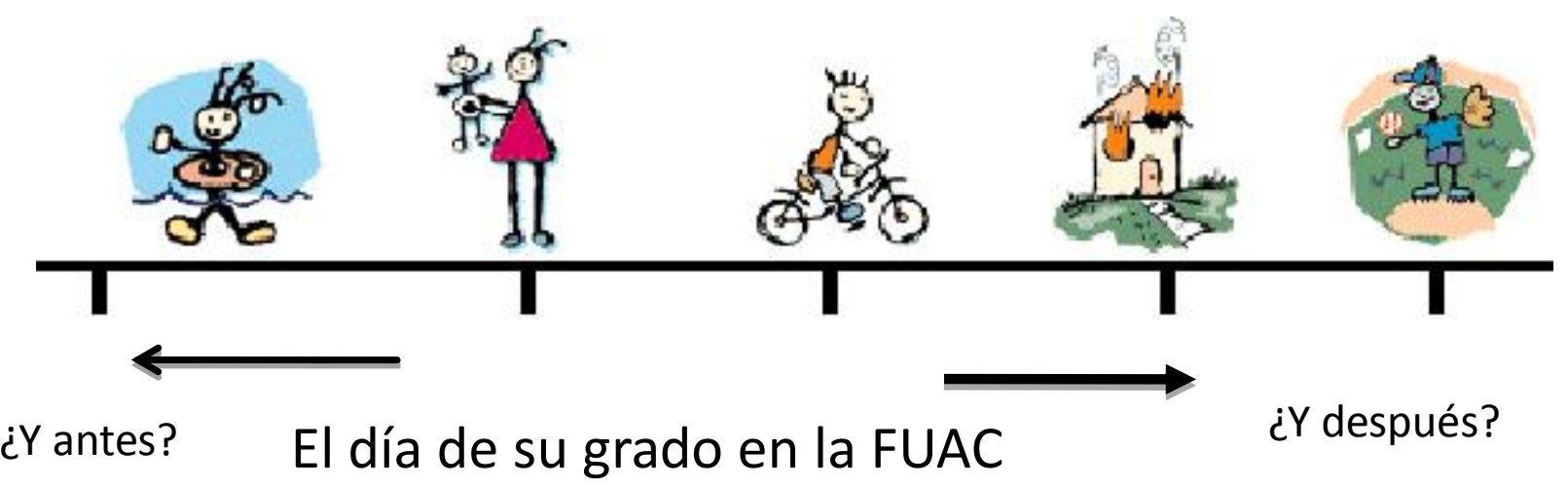

Formar una comunidad es tener la oportunidad de encontrarse, y los encuentros son un espacio para la memoria. La universidad los ha invitado el día de hoy para fortalecer los lazos comunitarios. Buscamos construir una comunidad de egresados que funcione como una red en la que construyamos un proceso, con la participación abierta de todos; una red para compartir ideas, aprendizajes y beneficios. Este es el primer momento de esa red a la que lo invitamos a sumarse.

El día de hoy implica un ejercicio para nuestra memoria autobiográfica, ya que estamos reconstruyendo el proceso que han tenido después de haber culminado su formación en la maestría. Conocer las transformaciones, los retos, las dificultades que han tenido, permite apreciar cómo nuestra red puede mejorar. El día de hoy, entonces, se considera un ejercicio para reconstruir esa memoria, pero también, en los próximos días recibirán un pequeño formulario que facilitará rehacer dicha memoria y, de forma anónima, le invitaremos a contestarlo. En él, reuniremos su proceso después de la maestría.

Así que bienvenidos al día de hoy. Para iniciar este ejercicio de la memoria, solo pensemos en nuestra vida como si fuera una línea del tiempo que atraviesa el espacio. Viajemos al pasado: ¿recuerdan el día de su grado?, ¿qué sucedió? , ¿recuerda el día que se matriculó? Bueno, ¿por qué lo hizo, qué lo motivó? Y ¿qué ha pasado después de graduarse de esta maestría? ¿Qué ha significado este proceso en su vida personal y profesional? ¿Ha encontrado dificultades? Si se devuelve un poco en el pasado a este tiempo anterior, ¿qué recuerda de esto? 


\section{Reflexiones finales}

Son varios los aspectos por considerar a la hora de planear y estructurar un proyecto de investigación y este no fue la excepción, pues a medida que sus integrantes se reunían, sus ideas encontraban no solo un interlocutor válido, sino que se articulaban de manera coherente y cohesionada a un mismo propósito. Cabe destacar también los elementos del trabajo cooperativo y colaborativo que siempre estuvieron presentes en las fases de diseño y planeamiento de la investigación; aspectos que fueron abordados en este escrito.

Esta dinámica no solo generó excelentes relaciones humanas en el equipo de trabajo, sino también, un espacio para la confrontación de ideas y el enriquecimiento conceptual; una oportunidad para reflexionar y analizar perspectivas, formas de pensar y posiciones distintas, dando cabida a la diferencia, al disenso y a la diversidad, pero también al encuentro de punto comunes, al acuerdo y al respeto y valoración del otro y de los demás. Todo esto fue de capital importancia en el replanteamiento y reestructuración de algunos aspectos, como por ejemplo, las categorías y elementos por considerar en los instrumentos, en la organización del encuentro de egresados y en la misma forma de trabajo y de interacción abordada por el colectivo para sus reuniones. Esta dinámica de trabajo hace que las sesiones se tomen más tiempo de lo acordado inicialmente.

Conviene resaltar también el diseño metodológico, el cual fue cuidadosamente ideado y esquematizado, pues los gestores del proyecto tienen campos disciplinares y perspectivas investigativas diferentes; sin embargo, en el apartado denominado Posible ruta metodológica se muestran algunos aspectos y se infieren otros, justifican los acuerdos establecidos que le dan identidad investigativa propia al proyecto. Para cada una de las fases de la ruta metodológica se establecieron protocolos, los cuales son una forma de organizar las actividades y de darles seguimiento y evaluación.

Es claro que un proyecto investigativo, como el que se expone de manera sucinta en este texto, exhorta a mirarnos en medio de las debilidades y fortalezas como miembros de un equipo de trabajo. También concientiza y reflexiona, de manera constante, en la relevancia, necesidad e impacto del desarrollo del proyecto para la comunidad del programa de Maestría en Didáctica de las Ciencias.

El equipo de trabajo asumió este proyecto investigativo como una oportunidad para visualizar el trabajo desarrollado a lo largo de la vida académica del programa de la Maestría en Didáctica de las Ciencias de la Universidad Autónoma de Colombia y como un espacio para socializar los productos investigativos realizados por los egresados, así como para 
generar un espacio de intercambio de experiencias docentes, lo cual posibilita la creación de sinergias en torno al desarrollo de la Didáctica de las Ciencias.

En ese sentido, la meta es la significación de la red de egresados como una práctica de una comunidad de aprendizaje permanente, pues los autores consideran que el conocimiento acumulado, transformado y reestructurado por las prácticas y contextos de los egresados son un potencial de aprendizaje colectivo invaluable para definir horizontes, problemas y campos de trabajo para el propio desarrollo de los docentes y la orientación del programa.

La generación de una red de egresados se orienta como parte de una política de fomento de la investigación con sentido social, pues de esta forma se contribuye al conocimiento, entendido como una construcción organizada en estructuras y dinámicas colectivas. De esta manera, una comunidad de práctica no solamente articula conocimiento, sino que se convierte en un espacio en el cual se generan procesos de relación profesional, diálogo e interacción multidisciplinar. Las comunidades de práctica significan, en el sentido más profundo; la generación del tejido comunitario en torno a problemáticas relevantes en el contexto social e institucional, propio del programa de Maestría en Didáctica de las Ciencias y de la universidad, en conjunto.

Una comunidad de práctica es, entonces, un principio generador de red de conocimiento $\mathrm{y}$, por ello, se fundamenta en el reconocimiento de la singularidad, la diversidad y la comunicación (Page, 2008; Blackmore, 2010). La singularidad, debido a que una comunidad de práctica encuentra en las diferencias de talentos y habilidades la mejor manera de construir inteligencia colectiva. Del mismo modo que la singularidad es, a la vez, el respeto por la diferencia de cada uno y de la diversidad de pensamiento que se articula gracias a la complementariedad de los sujetos, trayectorias de vida y competencias profesionales. Por su parte, en cuanto a la comunicación, porque implica la posibilidad de hacer circular el conocimiento y socializarlo, manera en que se hace posible el tejido colectivo que estructura la vida comunitaria que debe fomentar la universidad contemporánea.

\section{Referencias}

Baets, Walter. (2010). Knowledge Management and Management Learning: Extending the Horizons of Knowledge-Based Management. New York: Springer.

Bartholomew, David. (2008). Building on Knowledge: Developing Expertise, Creativity and Intellectual Capital in the Construction Professions. Singapore: Wiley-Blackwell. 
Bingham, Tony y Conner, Marcia. (2010). The New Social Learning. San Francisco, USA: Berrett-Koehler Publishers.

Blackmore, Chris. (2010). Social Learning Systems and Communities of Practice. Londres: Springer.

Bueno Campos, Eduardo. (2003). Gestión de Conocimiento en Universidades y Organismos públicos de Investigación. Madrid: Ed. Universidad Autónoma de Madrid.

Castells, Manuel. (2005). The Rise of the Network Society: Information Age: Economy, Society, and Culture (Vol. 1). Singapore: Wiley-Blackwell.

Geisler, Robert, Rauch, Gerd-Jörg, Geiger-Rudolph, Silke, Albrecht, Andrea, van Bebber, Frauke, Berger, Andrea ... Nüsslein-Volhard, Christiane. (2007). Large-scale mapping of mutations affecting zebrafish development. BMC Genomics, 8(11). doi:10.1186/1471-2164-8-11

Hagel, John, Seely Brown, John y Lang Davison. (2010). The Power of Pull: How Small Moves, Smartly Made, Can Set Big Things in Motion. New York: Basic Books.

Jong, Ton, Gog Tamara Van, Jenks, Kathleen, Manlove, Sarah, Hell, Janet van, Jolles, Jelle ... Boschloo, Annemarie. (2009). Explorations in Learning and the Brain: On the Potential of Cognitive Neuroscience for Educational Science. New York: Springer.

Koshy, Valsa. (2005). Action Research for Improving Practice. Londres: Paul Chapman Publishing.

Mahmoud, Qusay H. (2007). Cognitive Networks: Towards Self-Aware Networks. Canada: University of Guelph.

Mclntosh, Paul. (2010). Action Research and Reflective Practice: Creative and Visual Methods to Facilitate Reflection and Learning. New York: Routledge.

Page, Scott E. (2008). The Difference: How the Power of Diversity Creates Better Groups, Firms, Schools, and Societies. Princeton, NJ: Princeton University Press.

Pagel, Mark. (2012). Wired for Culture: Origins of the Human Social Mind. New York: W.W. Norton \& Company.

Thomas, Douglas y Seely Brown, Jhon. (2011). A New Culture of Learning: Cultivating the Imagination for a World of Constant Change. USA: CreateSpace Independent Publishing Platform.

Visser, Jan y Visser-Valfrey, Muriel. (2010). Learners in a Changing Learning Landscape: Reflections from a Dialogue on New Roles and Expectations. New York: Springer. 